\title{
L'INVESTISSEMENT DANS LA LITTÉRATIE : IDENTITÉS SOCIALES ET CAPITAL SYMBOLIQUE
}

Chiara Bemporad, Thérèse Jeanneret

Éditions de la Maison des sciences de l'homme | «Langage et société »

2016/3 $\mathrm{N}^{\circ} 157$ | pages 39 à 55

ISSN 0181-4095

ISBN 9782735120901

Article disponible en ligne à l'adresse :

https://www.cairn.info/revue-langage-et-societe-2016-3-page-39.htm

\section{Pour citer cet article :}

Chiara Bemporad, Thérèse Jeanneret« L'investissement dans la littératie : identités sociales et capital symbolique », Langage et société 2016/3 (N¹57), p. 39-55. DOI 10.3917/ls.157.0039

Distribution électronique Cairn.info pour Éditions de la Maison des sciences de l'homme.

(C) Éditions de la Maison des sciences de l'homme. Tous droits réservés pour tous pays.

La reproduction ou représentation de cet article, notamment par photocopie, n'est autorisée que dans les limites des conditions générales d'utilisation du site ou, le cas échéant, des conditions générales de la licence souscrite par votre établissement. Toute autre reproduction ou représentation, en tout ou partie, sous quelque forme et de quelque manière que ce soit, est interdite sauf accord préalable et écrit de l'éditeur, en dehors des cas prévus par la législation en vigueur en France. Il est précisé que son stockage dans une base de données est également interdit. 


\title{
L'investissement dans la littératie : identités sociales et capital symbolique
}

\author{
Chiara Bemporad et Thérèse Jeanneret \\ École de français langue étrangère, Université de Lausanne \\ chiara.bemporadQunil.ch; therese.jeanneret@unil.ch
}

"Lorientation vers le futur est $[\ldots]$ un savoirfaire qui se cultive " J. S. Bruner Le développement de l'enfant. Savoir dire, savoir faire (1983: 258)

\section{Introduction}

Dans cet article, nous présenterons et analyserons certains enjeux liés à l'acquisition de littératies en langues étrangères, dans le cas d'adultes amenés à utiliser les nouvelles langues en question dans différentes situations sociales. En particulier, nous nous intéresserons à la manière dont ces personnes envisagent des projets liés à ces nouvelles langues (le français ou l'espagnol) en mettant en évidence les liens entre les projets tels qu'ils sont présentés et formulés et les relations de pouvoir que ces personnes vivent ou ont vécues. En d'autres mots, nous montrerons comment les identités sociales expérimentées influent sur les désirs et les projets liés à ces langues, c'est-à-dire sur l'investissement de ces personnes envers ces langues.

En nous appuyant sur les travaux de Norton $(1995 ; 2013$, notamment), nous définirons cet investissement vers l'acquisition de littératies dans de nouvelles langues comme une construction discursive (Schütz 1987) élaborée par une personne qui manifeste une connaissance du monde en établissant des liens entre ses pratiques langagières et les positionnements sociaux attendus dans les univers liés à ces langues. Norton fait en effet de l'investissement une élaboration de la personne dans 
une situation donnée: elle conçoit ainsi la relation que cette dernière établit avec la langue qu'elle s'approprie comme labile, comme variant en fonction des positionnements sociaux expérimentés, des liens entre ces positionnements et ses pratiques langagières et des circonstances où l'investissement est relaté.

Ainsi, l'appropriation de la littératie dans une nouvelle langue par une personne, les situations sociales que cette dernière expérimente, les identités qu' elle est amenée à endosser forment une dynamique qui influe sur son investissement dans cette appropriation: les expériences sociales orientent les investissements de la personne, tandis que se transforment les images qu'elle se fait d'elle-même, de ses projets, etc.

Pour étudier l'investissement envers ces deux langues étrangères, nous nous intéresserons à ce que les personnes disent de leurs lectures et plus généralement de leurs pratiques littératiées, des raisons pour lesquelles elles ont mis l'accent sur ce versant de leur appropriation et de ce qu'elles en attendent en termes de positionnements sociaux. Nous ne concevrons pas les pratiques littératiées investies (c'est-à-dire relatées et projetées) seulement comme des observables langagiers, mais également comme des conduites incluant des attitudes, des émotions, des représentations et des valeurs (Barton et Hamilton 2010). En effet, comme Bourdieu (1982 notamment) l'a relevé, si toute pratique langagière s'inscrit dans un champ de forces en tension entre différentes formes de pouvoir symbolique, les pratiques écrites s'y insèrent de façon spécifique. Au moment de l'entrée dans la culture (Bruner 1996) et à travers l'apprentissage de l'écrit, déjà dans la première langue, la personne entre en contact avec la valeur symbolique (donc aussi sociale et économique) que cette culture attribue à ces pratiques littératiées et à leur apprentissage. L'analyse de ces pratiques ne peut ainsi faire l'économie des dimensions de pouvoir et de domination sociale (voir les New Literacy studies présentées par Fraenkel \& Mbodj 2010, notamment). C'est pourquoi il importe de prendre en compte la valeur attribuée aux pratiques littératiées dans de nouvelles langues pour comprendre et analyser les comportements discursifs des personnes plurilingues dans différents contextes sociaux, ainsi que l'ont relevé des travaux récents relatifs au rapport entre littératie et plurilinguisme, comme Hornberger \& Skilton-Sylvester (2000). Dans ce sillage, Molinié \& Moore (2012) et Dagenais (2012) soulignent également à quel point les pratiques littératiées « sont inextricablement liées aux structures de pouvoir dans les milieux où elles sont situées [et] sont interprétées de façons différentes par les groupes sociaux [...] en fonction des conditions matérielles et sociales en place" (Dagenais 2012 : 19). 
L'objet de notre étude concernera donc le lien entre les discours sur les pratiques littératiées et leur appropriation d'une part, et la valeur symbolique et sociale qui leur est attribuée d'autre part. Nous verrons en effet que c'est cette valeur, appréhendée en termes de capital symbolique et langagier, qui détermine l'investissement des personnes.

En conformité avec cette conception, les données qui serviront à discuter cette question sont des extraits d'entretiens recueillis par Bemporad (à paraitre) auprès de trois étudiantes de l'École de français langue étrangère de l'Université de Lausanne: Alessia (une étudiante suisse italophone), Helen et Sophie (deux étudiantes canadiennes anglophones). Ces données comprennent des autobiographies écrites de lectrices plurilingues (Bemporad 2010 et 2012) et des entretiens oraux effectués à la suite de ces textes ${ }^{1}$. Les premières sont des textes écrits, dans lesquels les étudiantes retracent un bilan de leurs trajectoires de vie par rapport aux langues qu'elles ont apprises et à leurs lectures dans ces différentes langues. Ces textes portent sur leurs représentations des langues à apprendre, leurs difficultés et succès, les rencontres et événements qui les ont poussées à faire des choix de vie impliquant l'appropriation de différentes pratiques littératiées dans différentes langues étrangères. Ils permettent un accès aux imaginaires liés aux langues des informatrices et traitent ainsi notamment de leur investissement vis-à-vis de ces langues à apprendre.

Dans cette étude, nous sélectionnons des extraits des données qui portent plus particulièrement sur l'investissement dans la lecture et l'étude de certains textes à appréhender dans ces langues, textes qui, en tant que textes littéraires, par exemple, jouissent pour la plupart d'une reconnaissance sociale. Il s'agit donc d'une recherche qui privilégie le point de vue émique de la personne et qui base sa rigueur méthodologique sur l'analyse discursive (forme et contenu) des discours produits (Baroni et Jeanneret 2008). Comme l'indique Deprez (1994 : 40), « les propos tenus sur la réalité ont aussi leur réalité: on ne peut pas indéfiniment rejeter l'imaginaire (le dicible) au profit du réel (l'observable), ni les opposer ».

Dans les extraits choisis, nos informatrices expliquent le sens et la valeur qu'elles donnent à leurs pratiques littératiées dans leurs différentes langues en revenant sur les circonstances qui ont entouré leur initiation à ces pratiques ainsi que sur la manière et les raisons pour lesquelles elles prévoient

1. La recherche concerne une étude longitudinale (se poursuivant entre six mois et deux ans) auprès de douze informateurs et informatrices, tous étudiant à l'École de français langue étrangère de l'UNIL. Ici seules les données discursives de trois étudiantes seront utilisées. 
de les poursuivre. Ils permettent de repérer la conception des pratiques littératiées de nos trois informatrices, conception qui détermine leur investissement, dans le sens où elles nous expliquent ce qu'elles attendent de leurs différentes pratiques langagières en termes d'adéquation aux normes sociales des milieux auxquels elles souhaitent appartenir. En l'occurrence, il s'agit plus particulièrement de leur lecture d'œuvres littéraires en français et en espagnol et de leur maitrise progressive des pratiques attachées à ces lectures (commentaires littéraires, exposés oraux, lecture de textes critiques, etc.).

\section{Le marché linguistique comme orientation de l'investissement}

L'analyse que nous faisons de ces données s'appuie sur certains éléments centraux du modèle bourdieusien de l'économie des biens symboliques (notamment Bourdieu 1982). En particulier, nous rattachons les différentes formes d'investissement décrites par nos informatrices à la manière d'accéder, grâce à leur appropriation langagière et culturelle, à un capital symbolique qui soit reconnu par les réseaux sociaux auxquels elles aspirent à appartenir. Ce capital prend différentes formes selon les contextes sociaux et c'est la manière dont les informatrices comprennent et adhèrent à telle ou telle conception de capital symbolique qui conditionne leur investissement dans différentes pratiques de lecture.

Le choix d'étudier l'investissement envers le développement des pratiques littératiées dans différentes langues met en évidence la façon dont nos informatrices analysent le marché des biens linguistiques et symboliques qu'elles expérimentent. L'orientation qu'elles donnent à leur investissement peut être saisie à travers les différentes formes de capital symbolique. Ainsi, nos données illustrent une description et une adhésion aux formes de capital culturel telles que les a proposées Bourdieu (1979) : capital à l'état incorporé (c'est-à-dire intériorisé sous forme d'habitus) et capital à l'état objectivé, c'est-à-dire relatif aux biens culturels. Nous verrons également que d'autres conceptions du capital apparaissent aujourd'hui, qui suivent une logique de marché différente.

Nos analyses requièrent donc l'articulation de ces deux conceptions du capital symbolique. En effet, grâce à la diversité de leurs socialisations, ces trois étudiantes ont intériorisé les valeurs sociales attribuées aux usages linguistiques et plus particulièrement aux pratiques littératiées. En même temps, elles identifient les zones de leur répertoire langagier qu'elles ont intérêt à élargir pour avoir accès à tel ou tel pouvoir langagier (ce que Bourdieu 2001 : 113 désigne par "l'anticipation des profits»).

L'investissement dans l'appropriation de la langue étrangère s'oriente donc vers un ensemble de réponses aux positionnements sociaux expé- 
rimentés, subis et souhaités. Dans ce sens, il sous-tend des actions transformatrices qui l'ancreront dans des projets d'appropriation concrets, ce qui a été décrit en termes de capacité d'action ou agentivité (agency) et qui permet d'expliquer la possibilité de résister à la reproduction et de subvertir l'habitus (Sterponi et Bhattacharya 2012). Nous utilisons cette approche constructiviste pour mettre en évidence dans nos données la fonction médiatrice de l'investissement qui, à travers le sens et la valeur qu'il manifeste pour la personne, donne forme à ses pratiques. Nous verrons ainsi que c'est l'investissement qui détermine la manière dont les informatrices décrivent leurs pratiques littératiées, leurs modes de déploiement et de manifestation.

En cohérence avec cette conception de l'investissement, nous nous proposons de traiter l'appropriation d'une langue étrangère comme sous-tendant une participation de la personne à différentes pratiques sociales. En effet, bien que l'on ait longtemps appréhendé la rencontre d'une personne et d'une langue à travers la métaphore du bain linguistique - dans laquelle l'accès à la langue est vu comme la rencontre d'une personne, d'un contexte (pourvoyeur d'informations parallèles ou donnant explicitement le sens) et d'une chaîne sonore ou graphique - nous mettrons l'accent, pour notre part, sur la participation de la personne à la production du discours. Loin d'être prioritairement une réceptrice, la personne devient alors une actrice des discours qui lui donnent accès à la langue par les activités qu'elle réalise (Kern et Liddicoat 2008).

Cette affirmation est centrale dans les conceptions actionnelles ou praxéologiques de l'appropriation. Ce sera alors l'ensemble des médiations sémiotiques de ces activités qui représentera, pour la personne, ses accès au discours dans la langue étrangère. La part langagière des activités peut être centrale ou marginale: elle peut, seule, permettre l'achèvement de l'activité ou ne jouer qu'un rôle minimal de régulation, qu'une mimogestualité peut à la limite remplacer. Dans ses débuts dans la pratique d'une langue étrangère, la personne sera impliquée plus facilement dans des activités " peu » verbales, et très contextualisées ${ }^{2}$ (pour saisir la diversité et la subtilité des ajustements réciproques lors d'un échange communicationnel intercommunautaire sans aucune langue commune, voir Noyau 1984).

2. Les associations s'intéressant aux personnes immigrées savent bien utiliser le bricolage, la couture et la cuisine pour entrer en contact avec des personnes avec lesquelles elles ne partagent aucune langue (voir pour un exemple le film L'apiculteur - Der Imker de Mano Khalil). 
Cette conception de l'accès aux discours en tant que médiation sémiotique des activités sociales influence la conception de l'appropriation d'une langue étrangère en ce qu'elle la lie aux modes de relations que les personnes ont entre elles et avec des objets culturels, plus spécifiquement, aux types d'activités symboliques et sociales qu'elles sont en situation de réaliser et en conséquence aux investissements qui y sont liés. Elle invite à considérer l'appropriation comme une socialisation langagière (Duff, $2007,2008)$ qui se réalise à la fois dans et par la diversité des interactions sociales dans lesquelles est impliquée la personne.

Ainsi, la manière dont la personne se représente son appropriation est dépendante des modes de liaison entre ses pratiques langagières, les assignations sociales qu'elle expérimente et à la valeur qu' elle leur attribue: elle en conçoit des projets d'appropriation dans différents domaines qui sont intrinsèquement liés à ses expériences sociales dans les champs qu' elle rencontre et dans lesquels elle s'investit. C'est de cette manière que naissent, vivent et se maintiennent les différentes formes d'investissement pour la langue à apprendre.

Dans nos données, l'investissement est le plus souvent contemporain des expériences de socialisation (puisque nos informatrices vivent à ce moment-là en Suisse romande), mais la dynamique qui s'établit entre l'investissement et les expériences de nos informatrices et qui prend appui sur leur passé s'oriente également vers l'avenir puisque les projets de nos informatrices jouent un rôle dans le sens qu'elles donnent à leurs pratiques littératiées.

C'est ainsi que l'investissement d'une personne dans ses pratiques langagières est tributaire de la manière dont elle comprend sa relation au monde social et représente donc un élément de son identité (Zeiter 2015). Comme nous le montrerons dans des extraits de nos entretiens, une dynamique se met en place entre la personne, ses pratiques langagières, les investissements qu'elle expérimente, le sens de qui elle est, c'est-à-dire sa subjectivité dans les termes de Norton, à la suite de Weedon (1987), et la manière dont son identité se construit.

\section{Pratiques littératiées et capital culturel}

Ce qui, à nos yeux, permet d'analyser nos données en terme d'investissement, donc comme une manière de lier un projet d'appropriation à une amélioration de l'acceptabilité sociale, tient à la relation que les personnes établissent entre les compétences dans la langue qu'elles souhaitent développer et le "profit de distinction" (Bourdieu, 2001) qu'elles en attendent. 
Pour que nos informatrices puissent produire les extraits de discours que nous reproduisons ici, il faut en effet qu'elles aient compris que c'est en tant qu'elle permet d'acquérir une distinction supplémentaire qu'une compétence devient un capital. Il faut ensuite qu'elles s'investissent qu'elles en émettent l'intention - dans l'acquisition de la compétence en question. Comme l'a montré Zarate (2000), le capital plurilingue est fonction de ses modes d'acquisition. La prégnance de l'apprentissage scolaire et universitaire chez nos informatrices est évidente: il va de soi que les modes d'investissement étudiés dans cet article y sont étroitement liés ainsi d'ailleurs qu'au contexte de recueil des données étudiées. Nos exemples renvoient également à des jeux de relations et de pouvoir liés à nos propres positionnements de chercheuses et d'enseignantes à l'université. À notre sens, cela doit être pris en compte pour une analyse compréhensive (Blanchet 2011) de nos données.

Ainsi, chez les trois étudiantes, l'investissement opéré devrait leur permettre de se donner le droit d'intervenir à bon escient dans des univers sociaux où est exigé un certain type de compétence, ainsi qu'on le voit chez Alessia qui, au moment de l'entretien, suit un Master en françaislangue étrangère et espagnol. En expliquant les raisons qui l'ont amenée à choisir d'étudier l'espagnol à l'université, alors que cette langue fait partie de son patrimoine familial (sa mère est Mexicaine), elle affirme en effet:

Exemple 1, (BL) $)^{3}$ : Alessia:

...avant de commencer la littérature espagnole ici parce que je me disais bon il faut que je reprenne l'espagnol parce que je souvenais pas de beaucoup des choses je savais parler de choses de la vie quotidienne je savais dire qu'est-ce que j'ai fait hier qu'est-ce que j'ai mangé et des choses basiques mais je savais pas parler de je sais pas faire un discours un peu plus élevé alors c'est pour ça que j’ai commencé à lire.

Dans ce discours, on reconnaît un investissement orienté vers une compétence " distincte et distinctive », ce qu'Alessia désigne par l'expression un discours un plus élevé, à l'image de ce qui dit Bourdieu (2001 : 88):

[...] il faut distinguer entre le capital nécessaire à la production d'un parler ordinaire plus ou moins légitime et le capital d'instruments d'expression (supposant l'appropriation des ressources déposées à l'état objectivé dans les bibliothèques, les livres et en particulier les "classiques ", les grammaires, les dictionnaires) qui est nécessaire à la production d'un discours digne d'être publié, c'est-à-dire officialisé.

3. Les données discutées sont étiquetées BL quand elles sont tirées des biographies de lectrice, et $\mathrm{E}$ quand elles sont tirées des entretiens oraux postérieurs dans lesquels $\mathrm{C}$ indique l'enquêtrice, $\mathrm{A}, \mathrm{S}$ ou $\mathrm{H}$ les informatrices. 
Dans le projet d'Alessia, l'appropriation des ressources littéraires en espagnol dispensées par l'université lui permettra ainsi de maîtriser de nouveaux instruments d'expression qui lui conféreront un prestige supplémentaire. Ces instruments d'expression relèvent du capital incorporé au sens de Bourdieu (1979). En effet, ils supposent un long travail d'acquisition qui coûte du temps, de l'énergie et des moyens économiques, et qui est donc " un travail du "sujet " sur lui-même ": Alessia s'investit pour se garantir un capital que Bourdieu définit comme " un avoir devenu être, une propriété faite corps, devenue partie intégrante de la "personne », un habitus" (1979: 4-5).

Alessia réalise ce travail d'intériorisation en accomplissant toute une série de démarches: elle s'inscrit à l'université, et étudie pendant plusieurs années en se passionnant pour la littérature espagnole. Son investissement commence toutefois par la conscience de la valeur sociale du livre en tant qu'objet, qu'elle " hérite » de sa famille mexicaine, famille d'intellectuels, journalistes et écrivains. Elle se souvient en effet, qu'au moment de déménager à Lausanne, elle amasse des livres qu' elle espère arriver à lire un jour:

Exemple 2, (E) : Alessia:

des fois mes cousins me donnaient des livres ou ma mère elle elle achète toujours des livres en espagnol alors la maison en était pleine de de livres en espagnol il y avait qu'à choisir alors [mm] et j'en ai encore mais je je il y a des livres que j’ai jamais touchés c'est en espagnol [mm] ici à Lausanne parce que moi j'étais toute contente ah oui maintenant je vais je vais essayer de lire en espagnol j'ai pris tous les livres que je voyais et je les ai je les ai mis dans dans ma dans ma valise je les ai remmenés ici et puis je les ai pas lus

Laction de mettre dans sa valise des livres est à la fois concrète et symbolique, elle met en évidence la valeur qu'Alessia attribue au livre en tant qu'objet avant de devenir moyen de développer son apprentissage. Alessia décrit ici un capital objectivé selon Bourdieu: le livre est vu d'abord dans sa "matérialité " et comme tel transmissible et même " transportable ". Même si ces livres-là ne sont pas devenus un capital incorporé (elle ne les a pas lus), l'action de les prendre avec soi, dénote le début de son investissement, qui se poursuit avec l'appropriation d'une langue distinctive et avec la lecture et l'étude de certains classiques de la littérature espagnole.

Dans un autre exemple, Alessia parle de son apprentissage du français, et de la volonté de reconduire le même parcours qu'en espagnol, avec l'étude de la littérature française. Elle manifeste ici aussi une conscience assez précise des conditions auxquelles ses pratiques seront reconnues et lui assureront donc un capital culturel. 
Exemple 3, (E) : Alessia :

À mais j'aimerais bien la découvrir [la littérature française] j'aimerais lire beaucoup plus en français [d'accord oui] ehm en français en littérature pas de textes des journaux ou de choses comme ça [oui oui] des livres des

$\mathrm{C}$ vous voulez vraiment des livres

À des classiques

C des classiques

À pour les connaître pour dire que je connais quel quelque chose de littérature française

Dans cet extrait, Alessia explique son désir de lire de la littérature française (j'aimerais lire beaucoup plus en français en littérature) à la fois par la connaissance qu'elle en tirera et par la reconnaissance que cette connaissance lui rapportera (pour dire que je connais quelque chose de littérature française). Ce faisant elle montre qu'elle a identifié la valeur que le groupe social qu'elle souhaite intégrer attribue aux savoirs sur la littérature et à sa pratique de lecture.

Ainsi, l'action envisagée sera transformatrice, dans le sens où Alessia cherche à infléchir en sa faveur les rapports de force symboliques et montre qu'elle est consciente des rapports de pouvoir que sous-tendent les pratiques langagières en général et le capital d'autorité que procurent la connaissance de la littérature et plus globalement les pratiques littératiées. L'investissement d'Alessia est ainsi tourné vers l'obtention d'un avantage social. On pourrait faire l'hypothèse que cette manière de concevoir l'investissement dans sa lecture oriente la manière de lire et de comprendre le texte.

Cet exemple permet également de souligner les liens entre investissement et image de soi. En tant qu'engageant la personne dans des actions transformatrices, l'investissement joue un rôle important dans les dynamiques identitaires. Ici, Alessia, en acquérant des compétences littératiées en français pourra développer une identité de personne cultivée dans cette langue et se faire reconnaître comme telle par les personnes habilitées à le faire.

On peut, à notre sens, ou considérer que l'investissement offre un soutien à la dynamique identitaire en cours ou le considérer comme partie prenante de cette même dynamique. En tant que configuration d'images de soi (Bourgeois 2006), la dynamique identitaire doit gérer des tensions et des écarts: l'investissement dans une appropriation en tant qu'orientation des activités peut provisoirement permettre à la personne de réguler et d'intégrer les différentes versions de soi qu'elle expérimente. Pour Alessia, ce sont ses liens avec l'espagnol et l'hispanité en général qui ancrent un type d'investissement qu'elle pourra ré-activer pour le français. 
Dans le cas de Sophie, une étudiante canadienne anglophone ayant grandi en Colombie britannique dans une famille d'origine francophone, il y a tensions entre deux mondes de référence: le monde francophone européen, où elle étudie au moment du recueil de données et qui fait partie de sa conception de la francophonie, et le monde canadien, dans lequel elle a vécu jusque-là. D’une part, en effet, Sophie reconnaît la culture française dans ses aspects les plus élitistes:

Exemple 4, (E): Sophie:

[...] le français c'est la culture française c'est vraiment c'est plus important que les autres et puis ils contrôlent la langue comme on dit parce que l'académie français et tout ça. oui je crois que c'est plus. ils disent que c'est plus important de faire la littérature française pour ces raisons-là

D'autre part, elle cherche un compromis qui lui permettra de concilier ses deux identités:

$S \quad[\ldots]$ je crois que c'est important de faire les deux [littérature française et canadienne] parce que c'est vraiment l'histoire de la langue un petit peu et si on fait la littérature des Lumières et de la France avant et puis oui c'est important de faire la la la littérature canadienne je crois

C parce que vous êtes canadienne ou parce que..

$S$ parce que je suis canadienne

Dans le discours de Sophie, nous pouvons ainsi analyser une conception du marché linguistique structuré par le champ littéraire, selon un modèle européen traditionnel tel qu'il a été décrit par Bourdieu. Ses raisons de s'investir dans l'appropriation d'un capital culturel paraissent ainsi proches de celles d'Alessia. Par ailleurs, pour Sophie, être Canadienne impose la connaissance d'une littérature autre, la littérature canadienne. Ce double investissement remplira alors des fonctions différentes: le premier assurera le capital culturel nécessaire pour intervenir dans les univers sociaux qu'elle veut intégrer - et notamment le monde académique - le second assurera un pan de l'image de soi essentiel, lié à la canadienneté.

Ces exemples montrent que nos informatrices établissent un lien entre les pratiques littératiées auxquelles elles aspirent et leur positionnement dans les champs sociaux dans lesquelles elles fonctionnent. Elles construisent ainsi discursivement de manière pratique et compréhensive les analyses qu'elles opèrent de leurs expériences de socialisation.

\section{Pratiques littératiées et capital communicatif}

À côté de ces représentations qui s'analysent sans difficultés comme des investissements vers un capital culturel, nos données manifestent également des investissements vers d'autres formes de capital, plutôt communica- 
tif, adapté aux valeurs de nos sociétés néo-libérales, comme l'ont montré tant Heller et Boutet (2006) que Duchêne (2011). La mondialisation des échanges économiques requiert pour son fonctionnement des formes de capital linguistique qui mettent en avant l'efficacité communicative ainsi que des éléments culturels plus « ordinaires » tels que l'hexis corporelle, la maitrise de scripts communicatifs de base, la construction de représentations liées aux discours des médias, etc.

Comme ce capital est orienté vers l'utilitarisme et la communication plus immédiate, Heller et Boutet (2006) l'appellent capital communicatif. Déjà identifié par Gumperz et Cook-Gumperz (1982 : 4), ce capital devrait permettre de gagner un profit d'intelligibilité en ce qu'il permet d'assurer les échanges économiques, centraux dans les nouveaux processus de production et de consommation ${ }^{4}$.

The role communicative skills play has thus been radically altered in our society. The ability to manage or adapt to diverse communicative situations has become essential and the ability to interact with people with whom one has no personal acquaintance is crucial to acquiring even a small measure of personal and social control. We have to talk in order to establish our rights and entitlements. When we are at work we often rely on interactive and persuasive skills to get things done. Communicative resources thus form an integral part of an individual's symbolic and social capital, and in our society this form of capital can be every bit as essential as real property resources were once considered to be (Bourdieu 1973). (Gumperz \& Cook-Gumperz 1982: 4)

Ce capital communicatif est également perçu par nos informatrices. Par exemple, la seconde étudiante canadienne, Helen, qui a grandi en milieu anglophone, décrit comment, après avoir étudié le français à l'école dans un programme d'immersion française, sans investissement particulier, elle s'est décidée à reprendre des études en français en espérant y gagner un avantage matériel (tu peux avoir un emploi immédiatement après) :

Exemple 5, (E): Helen:

j'ai pris deux ans je pense que j'ai fait rien de français du tout mais l'année passée j'ai pensé mais si je deviens prof j'ai besoin d'une deuxième sujet et maintenant au Canada c'est très utile d'app- d'enseigner le français parce que tu peux avoir un emploi immédiatement après

La dynamique de l'investissement est ici clairement appuyée sur une logique d'utilité (c'est très utile). À ce propos, Heller (2011) a relevé comment au Canada on a assisté à un glissement dans les représentations sociales

4. En fait, tant Duchêne (2011) que Heller \& Boutet (2006) montrent que le profit d'intelligibilité n'est pas valorisé comme tel dans la nouvelle économie. 
des Canadiens pour lesquels, à la lumière des nouveaux changements économiques et sociaux, le français est passé, dans les discours, d'un « droit collectif " des communautés francophones minorisées à une langue perçue comme " bien d'échange sur le marché de la nouvelle économie mondialisée » de tous les Canadiens, ce que l'auteure nomme « français comme valeur ajoutée " (Heller 2011 : 13-14).

Cette conscience du français comme valeur ajoutée sur le marché du travail amène Helen à accomplir toute une série d'actions qui montrent comment son investissement suit la logique du capital communicatif. Dans sa biographie écrite de lectrice, elle affirme en effet:

Exemple 6, (BL) : Helen:

J'ai passé deux mois à Québec en but d'améliorer mon français. Quand j'étais là je suis allée à la libraire pour acheter un livre en français, j'étais vraiment motivée! Dans la libraire j’ai trouvé une section de livres traduits de l'anglais en français. Dans cette section il y avait un livre qui appartenait à une de mes séries préférées des livres écrits en anglais. Il s'appelait L'accro de shopping a Manhattan par Sophie Kinsella.

Quand j'ai commencé à lire feuilleter les pages de ce livre j’ai trouvé que c'était très facile à lire! [...] Le vocabulaire aussi est plus simple qu'un livre écrit par un francophone. [...] Je sais que c'est meilleur pour la culture de lire les livres écrits par les francophones, mais quelques fois le vocabulaire et l'orthographe des phrases écrits par les francophones sont trop difficiles à comprendre donc ce n'est pas amusant à lire du tout.

On voit que les arguments développés par Helen touchent à l'utilité du français d'une part, à l'efficacité de la lecture de littérature populaire traduite de l'anglais, d'autre part. Bien qu'Helen soit consciente de la valeur symbolique de la lecture d'œuvres en français (Je sais que c'est meilleur pour la culture de lire les écrits par les francophones), c'est une logique d'efficacité qui a primé sur la logique du capital culturel. Par ailleurs, comme dans le cas d'Alessia, son investissement commence par la considération du livre comme bien chargé d'une valeur qui est, au premier abord, plus matérielle et utilitaire que symbolique (elle va dans une librairie pour acheter un livre).

Cette identification du français comme opportunité d'employabilité (Lagarde 1996), ainsi que le pragmatisme mis en œuvre pour exercer les compétences en lecture peuvent représenter dans le même temps, une dépréciation du capital culturel et linguistique tel qu'il a été présenté jusqu'ici comme objet de l'investissement. Dans ce sens, les propos d'Helen sont très pertinents: au Canada, c'est probablement en tant que compétence de communication que le français constituera un capital. Comme le relèvent Heller et Boutet (2006 : 15) : 
[1] es transformations et mutations rapides des univers de travail, en ce qu'elles impliquent de plus en plus la reconnaissance, la valorisation et l'exploitation de cette ressource "naturelle " qu'est l'exercice du langage, construisent un paysage mondial dans lequel les langues, les répertoires verbaux, les compétences à communiquer deviennent autant d'enjeux économiques.

Ainsi nos données permettent-elles de voir une tension entre deux univers de référence: l'un, dominé par les valeurs culturelles véhiculées notamment dans les milieux académiques dans lesquelles gravitent les étudiantes, considère le capital symbolique comme un bien éducatif. L'autre, régi par les logiques de marché de la nouvelle économie que nos informatrices rencontrent dans les autres contextes sociaux qu'elles expérimentent y voit un bien économique. On peut relever ici que certains discours didactiques actuels (inspirés notamment par le Cadre européen commun pour les langues) participent à l'élaboration de ces logiques économiques qui fétichise la communication et le capital communicatif en mettant l'accent sur les activités qu'il faut pouvoir réaliser en langue étrangère mais en négligeant souvent les connaissances et compétences proprement linguistiques (et littéraires), nécessaires pour les réaliser (Maurer, 2011). Dans ce sens, nos informatrices sont en situation de percevoir que l'horizon d'interprétation sociale des habiletés langagières est multiple et parfois contradictoire, que le marché symbolique est orienté par des valeurs concurrentes qu'en étant qu'étudiantes en lettres elles expérimentent parfois dans leur contradiction, mais qu'elles semblent aussi capables de concilier.

C'est dans ce sens que l'investissement fonctionne comme médiation entre la personne et ses lectures: les pratiques littératiées sont investies à la fois pour accéder à des socialisations prestigieuses et pour améliorer qualitativement la compétence en langue étrangère. Bemporad (à paraître) montre d'ailleurs que les lectures different en fonction de ces deux orientations de l'investissement. Ainsi, les pratiques littératiées prennent-elles des formes différentes en fonction des situations envisagées: l'extrême diversité des usages que l'écrit régit, des plus exigeants aux plus courants, permet en effet une grande latitude dans les investissements.

\section{Conclusion. Dynamiques identitaires et capital symbolique plurilittératié}

Les discours de nos trois informatrices montrent que leur investissement pour les pratiques littératiées est lié à leurs projets de socialisations dans différents réseaux sociaux. Nos informatrices manifestent une analyse 
fine et pertinente des valeurs que les groupes sociaux qu'elles fréquentent (ou souhaitent fréquenter) accordent aux différentes pratiques langagières. Clairement, elles sont sensibles au prestige social lié à la connaissance de la littérature et plus généralement des pratiques littératiées, et sont prêtes à investir temps et énergie pour lire les grandes œuvres de la littérature française et/ou espagnole. Mais au moment où leur investissement se manifeste par un désir d'améliorer plutôt leurs pratiques langagières communicatives, elles sont prêtes également à user de lectures " clandestines " dans le monde universitaire.

On peut noter également que l'investissement dans les pratiques littératiées tel qu'il peut être décrit et analysé dans nos données a une double origine sociale: d'une part, il est clairement associé aux positionnements sociaux, d'autre part, il est objet de discours par l'effet du recueil de données. À ce titre, sa construction est médiée par l'interaction sociale entre l'informatrice et la chercheuse.

Par ailleurs, les investissements de nos informatrices ne sont pas le seul produit de stratégies mûrement réfléchies, délibérées. Les représentations qu'elles se font de leurs places dans les différents espaces sociaux à expérimenter, les identités qu'elles espèrent pouvoir incarner doivent être mises en rapport avec les positionnements sociaux déjà subis: l'investissement matérialise à la fois les possibilités de lutte pour des positions plus légitimes et l'exploitation de positions prestigieuses déjà occupées (Zarate 2000).

En liant l'investissement qu'une personne met en œuvre aux positionnements sociaux qu'elle expérimente ou s'attend à expérimenter, nous avons ainsi rencontré des investissements orientés vers l'acquisition de capitaux culturels bien différents, tournés vers une conception académique du rôle des langues dans la vie sociale (qu’on a défini comme capital linguistique et culturel) ou influencés par un pragmatisme induit également par le discours de la didactique (le capital communicatif). Cela a permis de mettre en évidence les dynamiques constituées par l'investissement dans les pratiques littératiées, les identités expérimentées dans les différents espaces sociaux et les projets de constitution d'un capital symbolique plurilittératié. 


\section{Références bibliographiques}

Baroni, R., et Jeanneret, T. (2008), « Parcours de vie, identités féminines et trajectoires d'apprentissage ", Langage et société 125, p. 101-124.

Barton, D. et Hamilton, M. (2010), "La littératie: une pratique sociale ", Langage et société 133 , p. 45-62.

Bemporad, C. (2010), « Pour une nouvelle approche de la littérature dans la didactique des langues: la biographie du lecteur plurilingue ", Bulletin Vals/Asla 91, p. 67-84.

- (2012), "Réflexivité, lecture littéraire et langues étrangères ", Babylonia 2, p. 31-36.

- (à paraître), Plurilinguisme et lecture littéraire. Une approche biographique pour la recherche et l'enseignement, Bruxelles, Peter Lang.

Blanchet, P. (2011). "Nécessité d'une réflexion épistémologique", dans P. Blanchet et P. Chardenet (éds), Guide pour la recherche en didactique des langues et des cultures: approches contextualisées, Montréal, Paris, Éditions des archives contemporaines, p. 9-19.

Bourdieu, P. (1979), « Les trois états du capital culturel ", Actes de la recherche en sciences sociales 130 , p. 3-6.

- (1982), Ce que parler veut dire: l'économie des échanges linguistiques, Paris, A. Fayard.

- (2001), Langage et pouvoir symbolique, Paris, Seuil.

Bourgeois, E. (2006), "La motivation à apprendre", dans E. Bourgeois et G. Chapelle (éds), Apprendre et faire apprendre, Paris, PUF, p. 229-246.

Bruner, J. S. (1983), Le développement de l'enfant. Savoir dire, savoir faire, Paris, PUF.

- (1996), L'éducation, entrée dans la culture: les problèmes de l'école à la lumière de la psychologie culturelle, Paris, Retz.

Dagenais, D. (2012), «Littératies multimodales et perspectives critiques", Les Cabiers de l'Acedle 9 (2), p. 15-46.

Deprez, C. (1994), Les enfants bilingues: langues et familles, Paris, CrédifDidier.

Duchêne, A. (2011), « Néoliberalisme, inégalités sociales et plurilinguisme: l'exploitation des ressources langagières et des locuteurs ", Langage et société 136 , p. 81-108. 
Duff, P. A. (2008), "Introduction to Volume 8: Language Socialization", in P. A. Duff and N. H. Hornberger (eds), Language socialization. Encyclopedia of Language and Education: volume 8, Springer, p. xxiii-xix.

- (2007), "Second Language Socialization as sociocultural Theory: Insights and Issues ", Language Teaching 40, p. 309-319.

Fraenkel, B. et Mbodj, A. (dirs) (2010), « Les New Literacy Studies, jalons historiques et perspectives actuelles ", Langage et société 133, p. 7-24.

Gumperz, J. J. and Cook-Gumperz (1982), «Introduction: Language and Communication of social Identity ", in Gumperz, J. J. (ed.), Language and social Identity, Cambridge, London etc., Cambridge University Press, p. 1-21.

Heller, M. et Boutet, J. (2006), «Vers de nouvelles formes de pouvoir langagier? Langue(s) et identité dans la nouvelle économie ", Langage et société 118 , p. 5-16.

Heller, M. (2011), «Du français comme "droit" au français comme "valeur ajoutée": de la politique à l'économique au Canada ", Langage et société 136, p. 13-30.

Hornberger, N. H., and Skilton-Sylvester, E. (2000), « Revisiting the Continua of Biliteracy: International and Critical Perspectives ", Language and Education 14 (2), p. 96-122.

Kern, R. et Liddicoat, A. (2008), «Introduction: de l'apprenant au locuteur/ acteur » dans C. J. Kramsch, D. Lévy et G. Zarate (éds), Précis de plurilinguisme et du pluriculturalisme, Paris, Éditions des archives contemporaines, 27-33.

Lagarde, C. (1996), Le parler "melandjao ", Perpignan, Presses universitaires de Perpignan.

Maurer, B. (2011), Enseignement des langues et construction européenne. Le plurilinguisme une nouvelle idéologie dominante, Paris, Éditions des archives contemporaines.

Molinié, M. et Moore, D. (2012), «Les littératies: une notion en questions en didactique des langues ", Les Cahiers de l'Acedle 9 (2), p. 3-14.

Norton, B. (2013), Identity and Language Learning, Bristol, Multilingual Matters. 
Norton Pierce, B. (1995), « Social Identity, Investment, and Language learning », TESOL QUARTERLY 29 (1), p. 9-31.

Noyau, C. (1984), « Communiquer quand on ignore la langue de l'autre", dans Noyau, C. et Porquier, R. (éds), Communiquer dans la langue de l'autre, Paris, Presses universitaires de Vincennes, 8-59.

Schütz, A. (1987), Le chercheur et le quotidien, Paris, Klincksieck.

Sterponi, L., et Bhattacharya, U. (2012), « Dans les traces de Hymes et audelà: les études de la socialisation langagière ", Langage et société 139, p. 67-82.

Weedon, C. (1987), Feminist practice and poststructuralist theory, Oxford, New York: B. Blackwell.

Zeiter A.-C (à paraître), Couples linguistiquement mixtes et appropriation des langues, Lyon, Éditions de l'École Normale Supérieure.

Zarate, G. (2000), «Constitution d'un capital plurilingue et économie d'une identité pluriculturelle: deux études de cas ", Mélanges RAPPEL 25, p. 75-89. 